\title{
FACTORES SOCIODEMOGRAFICOS INTRA Y EXTRA ORGANIZATIVOS RELACIONADOS CON SINDROME DE QUEMARSE POR EL TRABAJO EN PROFESIONALES DE SALUD DE HOSPITALES. ANCASH. PERU. 2011.
}

\author{
FACTORS SOCIODEMOGRAPHIC INTRA AND EXTRA ORGANIZATIONAL \\ RELATED TO BURNOUT SYNDROME IN HEALTH PROFESSIONALS IN \\ HOSPITALS. ANCASH. PERU.2011.
}

\author{
Rosario Yslado M. ${ }^{1}$, Rosa Atoche B., Maximiliana Quispe G., Ladmila Ruiz G., Jorge Medina G. \\ Universidad Nacional Santiago Antúnez de Mayolo, Huaraz, Perú
}

(Recibido el 04/10/2011, Aceptado el 28/11/ 2011)

\begin{abstract}
RESUMEN
Es una investigación descriptivacorrelacional, cuyo objetivo fue analizar y relacionar los factores sociodemográficos, intra y extraorganizativos, con el síndrome de quemarse por el trabajo (SQT) de médicos, enfermeros (as) y obstetras de cinco hospitales dela zona sierra de Ancash. La muestra fue de 127 profesionales nombrados, 54 médicos, 57 enfermeros y 16 obstetras, de ambos sexos, entre 25 a 69 años de edad.Se aplico el cuestionario síndrome de quemado por el trabajo en profesionales de la salud, DIRES Ancash - 2011, validado por criterio de jueces y estudio piloto, e Inventario Burnout de Maslach-MBI. Se encontró que la mayoría (78.0\%) de los profesionales presentan fase inicial delSQT, con bajo estrés; $2.4 \%$ se encuentran en la fase intermedia y el $19.6 \%$ en la fase final con alto estrés laboral crónico y desgaste profesional. Existen diferencias significativas entrelos grupos ocupacionales en relación a la dimensión baja realización personal,que en mayor porcentaje presentan los médicos. Asimismo hay diferencias significativas por género, los varones presentan enmayor frecuencia falta de realización personal en el personal. No se ha encontrado diferencias significativas para las dimensiones cansancio emocional y despersonalización. Se concluye que no existe asociación significativa entre los factores sociodemográficos, intra y extraorganizativos, con el SQT en todas sus fases; pero si hay relación significativa entre el factor intraorganizativo grupal (apoyo socioemocional, conflictos interpersonales con compañeros de trabajo, conflictos gremiales) y la fase final de SQT de los grupos ocupacionales citados.
\end{abstract}

Palabras clave: Síndrome de quemarse por el trabajo, factores sociodemográficos, factoresintra y extraorganizativos.

\footnotetext{
1 Profesora principal de la Facultad de Ciencias Medicas de la UNASAM. E-mail:ysladomx@yahoo.com.mx
} 
Factores Sociodemograficos Intra y Extra Organizativos Relacionados con Sindrome de Quemarse por el Trabajo en Profesionales de Salud de Hospitales. Ancash. Perú. 2011.

\section{ABSTRACT}

It is adescriptive correlational research, the aim was to analyzeand relatefactors sociodemographic, intra andextraorganizational, with syndromeof burningfor the work(SBW)) of doctors, nurses (as)and obstetriciansof fivehospitalsfromAncash. The samplewas 127appointedprofessionals, 54 doctors, 57 nursesand 16midwives, of both sexes, between 25 and 69years old. It was applied the questionnairesyndromeburnedby work inhealthcare,DIRESAncash -2011,validatedby criteriaof judges andpilot study,and MBI -MaslachBurnoutInventory. It was found thatthe majority(78.0\%) of health professionalshaveearly phaseof burnout, low stress, and $2.4 \%$ are in the intermediate phaseand $19.6 \%$ in the final phase,highstress chronic work and burnout. There are significant differencesbetweenoccupational groupsinrelation to the dimensionlow personal realization, thatdoctorshavea higher percentage.There arealsosignificant differences by gender, the male have moreoften low personal realizationat work. No were foundsignificantdifferences foremotional exhaustionand depersonalizationdimensions. In conclusion there isntsignificant associationbetween factorssociodemographic, intra andextraorganizationalwithburnoutin all its phases, but if there issignificant relationship betweenfactor intraorganizational group(support social-emotional, interpersonal conflicts withco-workers,guild conflicts) and final phase ofburnoutin the professionals groupsmentioned.

Keywords: Burnoutsyndromeby work,factors sociodemographic, factorsintraand extraorganizational.

\section{INTRODUCCION}

En el presente siglo, losprofesionales de la salud están expuestos a diferentes riesgos laborales de tipo físico, psicológico, químico y biológico y de manera especifica al Síndrome de Quemado por el Trabajo (SQT), conocido a nivel internacional y nacional como Síndrome de "burnout", es uno de los riesgos más importantes para este tipo de profesionales, lo cual ha sido demostrado científicamente a través de diferentes estudios realizados en el Perú y otros países. (Albaladejo, Villanueva, Ortega, Astasio, Calle y Domínguez, 2004; Albieri y Do Carmo, 2009; Aragón, Morazán, y Pérez, 2008; Arauco, 2007; Arauco, Enciso y García, 2004; Coronado, 2006; Gamonal, García y Silva, 2008; Gil-Monte y Moreno-Jiménez, 2007; Grau, Flichtentrei, Suñer, Prats y Braga, 2009; Hospital Nacional Arzobispo Loayza de Lima, 2010; López, Zegarra y Cuba, 2006: Llaja, 2002; Melita, Cruz y Merino, 2008; Mercado, 2006; Palmer, 2005; Peral y Vargas, 2009; Quiroz y Saco, 2003; Tapia et al., 2009).

Este constructo multidimensional también ha sido denominado síndrome del estrés laboral asistencial por Maslach y Jackson (1997), y comprende las dimensiones de agotamiento emocional, despersonalización y disminución de la realización personal y afecta principalmente a profesionales que prestan servicios asistenciales, que trabajan continuamente en contacto directo con personas que tienen problemas de salud física y/o mental y como tal estos profesionales se implican con los problemas de sus pacientes, y consecuentemente acumulan estrés crónico, que puede cansar emocionalmente y conducir a una situación de agotado o profesional quemado. 
Para fines de esta investigación se asumió de una parte, la perspectiva psicosocial propuesta por Maslach (1982), que considera el SQT como un proceso y entiende que un profesional esta quemado (agotado), cuando las demandas del entorno laboral, le han sobrepasado reduciendo su capacidad de adaptación. El estrés seria el resultado individual de un excesivo desbalance entre las demandas planteadas por la institución laboral y los recursos adaptativos con los que se cuenta. Si el desbalance es muy intenso o se mantiene demasiado tiempo, genera estrés crónico, que afecta la salud integral, la calidad y productividad laboral del profesional.

De otra parte, se ha considerado el modelo sobre el proceso del SQT de Golembiewski. Munzenrider y Carter (1983), que concluye que el SQT se desarrolla gradualmente a través de ocho etapas y tres fases (inicial, medio y final) y que progresa de despersonalización a baja realización personal en el trabajo, siendo el sentimiento de agotamiento emocional, el ultimo síntoma en establecerse e indicador de SQT alto.

El SQT se concibe como una respuesta de inadaptación frente al estrés laboral crónico, que se presenta en los profesionales de salud, que al laborar en establecimientos de salud del MINSA de la zona sierra de Ancash, estarían expuestos a ejercer su profesión en un sistema organizacional deficiente, en condiciones laborales difíciles, y con bajas remuneraciones, que en parte son factores condicionantes del problema de salud mental - ocupacional referido.

Los resultados encontrados revelan las características de las variables sociodemográficas, intra y extraorganizativas relacionadas con el SQT (fase final), como de los estresores laborales, factores de riesgo y grupos en riesgo presentes en el entorno y contexto laboral investigado. Los datos encontrados son insumos necesarios y relevantes para la implementación de estrategias de intervención (individual, interpersonal y organizacional) para la prevención y afrontamiento del SQT en todas sus fases, a fin de proteger y restablecer la salud física y mental de los trabajadores de salud, optimizar su desempeño laboral, promover entornos laborales saludablespara mejorar la calidad de atención en los servicios de salud, y lograr la revalorización de los servicios asistenciales en entidades publicas, cuyo prestigio esta cuestionado por la sociedad.

Se han revisado algunas investigaciones previas sobre SQT, a nivel internacional, como las de: Albaladejo, Villanueva, Ortega, Astasio, Calle y Domínguez (2004); Aranda, Pando, Salazar, Torres, Aldrete y Pérez, M.B. (2005), Palmer, Gómez, Cabrera, Prince, Searcy (2005); Albieri y Do Carmo (2009); Grau, Flichtentrei, Suñer, Prats y Braga (2009); Peralta y Vargas (2009). A nivelnacionalse han considerado los trabajos de: Quiroz y Saco (2003); Arauco, Enciso y García (2004); Dávalos (2005); Mercado (2006); López, Zegarra y Cuba (2006); Arauco (2007); Hospital Nacional Arzobispo Loayza de Lima (2010). A nivel local las que se mencionan a continuación:Abarca y Huamán (2004); Lugo (2010) y de Flores y Cueva (2011). 
Factores Sociodemograficos Intra y Extra Organizativos Relacionados con Sindrome de Quemarse por el Trabajo en Profesionales de Salud de Hospitales. Ancash. Perú. 2011.

\section{OBJETIVOS}

\section{General}

- Analizar los factores sociodemográficos, intra y extraorganizativos relacionados con el síndrome de quemarse por el trabajo de los médicos, enfermeros (as) y obstetras nombrados de cinco hospitales del Callejón de Huaylas, Ancash.

\section{HIPÓTESIS}

\section{General}

- Existen factores sociodemográficos, intra y extraorganizativos, que están relacionados con el síndrome de quemarse por el trabajo en los médicos, enfermeros (as) y obstetras nombrados de cinco hospitales de Ancash.

\section{METODO}

El presente es un estudio transversal, descriptivo correlacional-causal, de corte transversal - analítico.

La muestra ha sido de 127 profesionales nombrados, 54 médicos, 57 enfermeros y 16 obstetras, de ambos sexos, cuyas edades oscilaron entre 25 a 69 años de edad, pertenecientes a los hospitales de Recuay, Huaraz, Carhuaz, Yungay y Caraz, que participaron voluntariamente de acuerdo a una selección censal, con criterios de inclusión y exclusión.

\section{VARIABLES}

- Variable dependiente: Síndrome de quemado por el trabajo.

- Variable independiente: Factores sociodemográficos, intra y extraorganizativos.

\section{Instrumentos}

Se ha utilizado la técnica de encuesta y el cuestionario "Síndrome de quemado por el trabajo en profesionales de la salud de DIRES Ancash. 2011", que comprende tres partes: I. Aspectos sociodemográficos (06 ítems), II. Aspectos intra y extraorganizativos (36 ítems) y III. El Inventario Burnout de Maslach MBI (22 ítems), que mide el desgaste profesional, considerando tres dimensiones: cansancio emocional, despersonalización y disminución de realización personal; examina los sentimientos y actitudes del profesional en su trabajo y con respecto a sus pacientes. La administración puede ser individual o grupal, en un tiempo aproximado de 30 a 35 minutos.La validez del cuestionario", ha sido establecida mediante el análisis y comprobación de los reactivos a través de un estudio piloto y criterio de jueces. Para establecer la confiabilidad delInnventario "Burnout de Maslach-MBI instrumento, se utilizó el coeficiente alfa de Cronbach que fue 0.83 .

\section{RESULTADOS}

\section{Frecuencia y porcentaje de SQT en profesionales de salud}

En la tabla 1, se aprecia que la mayoría (78.0\%) de los profesionales de salud, se encuentran en la fase inicial del SQT, es bajo el nivel de estrés ocupacional, que 
normalmente presentan los profesionales que realizan servicio asistencial en salud; el $2.4 \%$, son proclives para presentar SQT, por encontrarse en la fase intermedia; y menos de la cuarta parte de la muestra investigada (19.6\%) presenta nivel alto de SQT, es decir se encuentran en la fase final del SQT. Asimismo se observa diferencias estadísticamente significativas $(p<0.05)$ entre las fases; el grupo que se encuentra en la fase inicial requiere acciones de prevención primaria y el grupo que se encuentra en la fase final requiere intervención asistencial (individual - grupal) y organizacional.

Tabla 1. Frecuencia y porcentaje de SQT en profesionales de salud, según las etapas de Golembiewski

\begin{tabular}{|c|c|c|c|}
\hline Etapa & Frecuencia & Porcentaje & Fase de SB \\
\hline I & 10 & 7,9 & \multirow{3}{*}{ Inicial (78.0\%) } \\
\hline II & 0 & 0,0 & \\
\hline III & 89 & 70,1 & \\
\hline IV & 2 & 1,6 & \multirow{2}{*}{ Intermedio (2.4\%) } \\
\hline $\mathrm{V}$ & 1 & 0,8 & \\
\hline VI & 2 & 1,6 & \multirow{3}{*}{ Final (19.6\%) } \\
\hline VII & 20 & 15,7 & \\
\hline VIII & 3 & 2,4 & \\
\hline Total & 127 & 100,0 & \\
\hline Chi-cua & arson $=338,14$ & G.L. $=6$ & $p<0.05$ \\
\hline
\end{tabular}

\section{SQT fase inicial en profesionales de la salud}

Lo descrito en la tabla 2, confirma la primera hipótesis específica. Se aprecia que los médicos, enfermeros (as) y obstetras nombrados de los cinco hospitales investigados, presentan con mayor frecuencia SQT en la fase inicial mayor a 70\%.

Tabla 2. Frecuencia y porcentaje de SQT fase inicial en profesionales de la salud de hospitales de Ancash.

\begin{tabular}{cccccc}
\hline \multirow{2}{*}{ FASE } & \multicolumn{3}{c}{ PROFESION } & Total \\
& & Medico & Enfermera & Obstetricia & \\
\hline Inicial & SI & 40 & 45 & 14 & 99 \\
& \multirow{2}{*}{$\mathrm{NO}$} & $74,1 \%$ & $78,9 \%$ & $87,5 \%$ & $78,0 \%$ \\
& 14 & 12 & 2 & 28 \\
& $25,9 \%$ & $21,1 \%$ & $12,5 \%$ & $22,0 \%$ \\
\hline \multirow{2}{*}{ Total } & 54 & 57 & 16 & 127 \\
& $100,0 \%$ & $100,0 \%$ & $100,0 \%$ & $100,0 \%$ \\
\hline
\end{tabular}


Factores Sociodemograficos Intra y Extra Organizativos Relacionados con Sindrome de Quemarse por el Trabajo en Profesionales de Salud de Hospitales. Ancash. Perú. 2011.

\section{SQT fase final en profesionales de la salud de hospitales de Ancash}

La segunda hipótesis específica se confirma parcialmente; los médicos presentan SQT en la fase final en $20 \%$. No así para el caso de las enfermeras y obstetras, cuyos porcentajes obtenidos han sido mayores. Sin embargo las diferencias no son significativas estadísticamente, pero si tendrían relevancia desde el punto de vista clínico. (Tabla 3).

Tabla 3. Frecuencia y porcentaje SQT fase final en profesionales de la salud de hospitales de Ancash.

\begin{tabular}{lccccc}
\hline \multirow{2}{*}{ FASE } & & & Profesión & Total \\
& & Medico & Enfermera & Obstetricia & \\
\hline \multirow{3}{*}{ Final } & \multirow{3}{*}{ SI } & 11 & 12 & 2 & 25 \\
& & $20.4 \%$ & $21.1 \%$ & $12,5 \%$ & $19.6 \%$ \\
& \multirow{2}{*}{ NO } & 43 & 45 & 14 & 102 \\
& & $79.6 \%$ & $78.9 \%$ & $87,5 \%$ & $80.4 \%$ \\
\hline \multirow{2}{*}{ Total } & 54 & 57 & 16 & 127 \\
& & $100,0 \%$ & $100,0 \%$ & $100,0 \%$ & $100,0 \%$ \\
\hline
\end{tabular}

\section{Dimensiones y etapas de SQT según grupo ocupacional}

En la tabla 4 se observa que no existen diferencias significativas entre los grupos ocupacionales con respecto a dos dimensiones (cansancio emocional, despersonalización), pues los valores de significación son mayores a 0.05 (significación teórica); sin embargo hay diferencias significativas entre los grupos ocupacionales en la dimensión de realización personal $(\mathrm{p}<0.05)$.

Tabla 4. Dimensiones de SQT según grupos ocupacionales

\begin{tabular}{cccccc}
\hline \multirow{2}{*}{ Dimensiones } & \multicolumn{2}{c}{ Promedio de Rangos por Grupos Ocupacionales } & \multicolumn{2}{c}{ Contraste } \\
\cline { 2 - 6 } & Médico(n=54) & Enfermera $(\mathrm{n}=57)$ & Obstetra $(\mathrm{n}=16)$ & X23 & Sig. \\
\hline $\begin{array}{c}\text { Cansancio } \\
\text { Emocional }\end{array}$ & 62,7 & 67,1 & 57,6 & 0,95 & 0,62 \\
\hline $\begin{array}{c}\text { Despersonalización } \\
\text { Realización } \\
\text { Personal }\end{array}$ & 66,2 & 61,2 & 66,7 & 0,61 & 0,74 \\
\hline
\end{tabular}




\section{Dimensiones y etapas de SQT según género de los profesionales de salud}

En la tabla 5, se observa que no existen diferencias significativas entre varones y mujeres con respecto a dos dimensiones (cansancio emocional, despersonalización), pues los valores de significación son mayores a 0.05 (significación teórica); sin embargo, hay diferencias significativas entre varones y mujeres en la dimensión de realización personal $(\mathrm{p}<0.05)$, el porcentaje es mayor para el caso de los varones, en comparación a las mujeres.

Tabla 3. Frecuencia y porcentaje SQT fase final en profesionales de la salud de hospitales de Ancash.

\begin{tabular}{ccccc}
\hline \multirow{2}{*}{ Dimensiones } & \multicolumn{2}{c}{ Promedio de Rangos } & \multicolumn{2}{c}{ Contraste } \\
\cline { 2 - 5 } & Masculino $(\mathrm{n}=53)$ & Femenino $(\mathrm{n}=73)$ & $\mathrm{Z}$ & Sig. \\
\hline Cansancio Emocional & 61,5 & 64,9 & $-0,52$ & 0,60 \\
\hline Despersonalización & 63,4 & 63,6 & $-0,03$ & 0,97 \\
\hline Realización Personal & 71,7 & 57,6 & $-2,14$ & 0,03 \\
\hline
\end{tabular}

\section{Relación entre SQT fase final y factor grupal}

El factor intraorganizativo grupal, está relacionado significativamente con el SQT en la fase final de los médicos, enfermeros (as) y obstetras nombrados, de los hospitales de Recuay, Huaraz, Carhuaz, Yungay y Caraz. (Tabla 6).

Tabla 3. Frecuencia y porcentaje SQT fase final en profesionales de la salud de hospitales de Ancash.

\begin{tabular}{lcccc}
\hline \multirow{2}{*}{ FACTOR } & \multicolumn{2}{c}{ FASE FINAL } & Total \\
& & SI & NO & \\
\hline \multirow{2}{*}{ Grupal } & No & 11 & 79 & 90 \\
& & $50,0 \%$ & $75,2 \%$ & $70,9 \%$ \\
& Si & 11 & 26 & 37 \\
& & $50,0 \%$ & $24,8 \%$ & $29,1 \%$ \\
\hline \multirow{2}{*}{ Total } & & 22 & 105 & 127 \\
& & $100,0 \%$ & $100,0 \%$ & $100,0 \%$ \\
\hline
\end{tabular}


Factores Sociodemograficos Intra y Extra Organizativos Relacionados con Sindrome de Quemarse por el Trabajo en Profesionales de Salud de Hospitales. Ancash. Perú. 2011.

\section{DISCUSION}

Al correlacionar estadísticamente las fases del SQT (inicial, medio y final) de los médicos, enfermeros (as) y obstetras nombrados de cinco hospitales dela zona sierra de Ancash, con los factores sociodemográficos, intra y extraorganizativos, se encontró que estas variables no se relacionan significativamente; por lo tanto no se ha confirmado la hipótesis general. Un resultado similar fue reportado por López, Zegarra y Cuba (2006). De otra parte al correlacionar algunos indicadores de las variables citadas, se encontró que existe relación significativa, entre el factor intraorganizativo grupal (apoyo sociolaboral en el trabajo, conflictos interpersonales con compañeros de trabajo, conflictos gremiales) y SQT en su fase final.

Los resultados hallados demuestran que con mayor frecuencia los médicos, enfermeros (as) y obstetras, presentan la fase inicial (etapa III) del SQT. La presencia de SQT en sus diferentes etapas y fases seria homogénea para los médicos y enfermeros(as), pues no se encontró significancia estadística según el hospital en el cual laboran. Estos resultados son coherentes con hallazgos en muestra de profesionales de la salud, que han sido reportados en el contexto internacional (Pérez y Vargas, 2009), y nacional (Arauco, Enciso y García, 2004; Dávalos, 2005; López, Zegarra y Cuba, 2006; Quiroz y Saco, 2003).

Si bien la prevalencia del SQT (fase inicial) es bajo para los médicos y enfermeros (as); sin embargo para el caso de los obstetras se encontró significancia estadística, es decir la presencia de SQT en sus diferentes fases no es homogénea, según el hospital en el cual laboran. Se observa mayor porcentaje de SQT fase inicial (III), $87.5 \%$, que en la fase final (VII) en los obstetras que trabajan en el hospital de Huaraz (Nivel II - 2); por lo tanto este grupo ocupacional estaría en mayor riesgo.

Se ha confirmado parcialmente la primera hipótesis especifica; es decir el 78.0\% de profesionales de la salud encuestados presenta estrés laboral mas que SQT propiamente dicho; siendo el mayor porcentaje para los obstetras, en segundo lugar para los enfermeros (as), y tercero para los médicos. De este resultado se infiere, que existen factores protectores intrínsecos o extrínsecos, que evitan la progresión a las siguientes fases del SQT (intermedio y final); sin embargo de no implementarse programas de prevención primaria y secundaria que permitan manejar el estrés laboral crónico, el nivel de SQT puede progresar hasta su fase final, caracterizado por desgaste emocional y profesional (Golembiewski, Munzenrider y Stevenson, 1986; Gil-Monte y Moreno-Jiménez, 2007).

Asimismo se encontró que solo el $2.4 \%$ de profesionales de la salud evaluados, se encuentra en la fase intermedia, y serian proclives para presentar SQT final. El $19.6 \%$ de médicos, enfermeros(as) y obstetras, presenta SQT en la fase final (etapas VI, VII, VIII), y específicamente los enfermeros (as) presentan $21.1 \%$, los médicos $20.4 \%$, seguido del grupo de obstetras (12.5\%); sin embargo las diferencias no fueron estadísticamente significativas, pero clínicamente si serian relevantes. Este resultado confirma parcialmente la primera hipótesis específica, que afirmaba la existencia de $20 \%$ de SQT (fase final) en los tres grupos ocupacionales. Estos resultadoscoinciden con la prevalencia de SQT alto para los 
enfermeros (as) y médicos, reportado en otros estudios internacionales, nacionales y regionales (Albieri y Do Carmo, 2009; Aranda et al, 2005; Dávalos, 2005; Flores y Cueva, 2011; Grau et al, 2009; Llaja, 2002; Palmer et al, 2005).

El resultado descrito en el párrafo anterior ha permitido identificar dos grupos ocupacionales en riesgo laboral psicosocial (enfermeros y médicos), con mayor desgaste profesional, propensos a presentar tanto enfermedades físicas, mentales, crónicas y agudas, deficiente calidad asistencial, renuncia prematura y otras consecuencias graves por SQT alto no tratado. Para el caso de los médicos los hallazgos descritos, son congruentes con los lineamientos teóricos desarrollados al respecto por Gálvez, Moreno-Jiménez y Mingote (2009).

Los profesionales de la salud en el siglo XXI, sea en el contexto nacional o regional,deben responder a demandas sociales, organizacionales y emocionales, exigidas por la naturaleza de su formación profesional, es decir deben atender diferentes problemas de salud, de manera oportuna, sin errores, con mística, y satisfacción, en inadecuadas condiciones laborales y con bajas remuneraciones, que no estaría acorde al esfuerzo desempeñado, ni gratificación esperada, generándose agotamiento físico, emocional y cognitivo; desarrollo de actitudes y sentimientos negativos hacia sus compañeros de trabajo y endurecimiento afectivo ante los pacientes, que puede a su vez ocasionar sentimientos de culpa; sentimientos de insatisfacción laboral, impotencia, desesperanza, perdida de entusiasmo y compromiso en el trabajo, como mayor percepción de reducida realización personal en su trabajo, en comparación a otros grupos ocupacionales.

También en este estudio se han encontrado diferencias significativas entre las fases inicial, intermedio y final del SQT; es decir, en mayor riesgo se encontrarían los portadores del SQT en la fase final, por lo tanto en este grupo seria urgente la intervención asistencial, interpersonal y organizacional, en cambio para los que se encuentran en la fase inicial seria pertinente implementar programas de prevención primaria y para los proclives (fase intermedia) seria adecuado programas de prevención secundaria.

Al comparar los valores obtenidos en las tres dimensiones del SQT, en la muestra estudiada (que en mayor porcentaje han sido del hospital de Huaraz, del servicio de cirugía, enfermeros (as), mujeres, en el rango de 50 a 59 años de edad, estado civil casados, con estudios de segunda especialidad, y casa propia), se observa que casi todos los profesionales de la salud (93.7\%), presentan un nivel bajo en la dimensión emocional- actitudinal de despersonalización, nivel alto en la dimensión cognitiva- emocional baja realización personal (89.8\%) y nivel bajo en la dimensión bioemocional-actitudinal cansancio emocional (79.5\%). Asimismo se encontró que no existen diferencias significativas entre los grupos ocupacionales con respecto a las dimensiones cansancio emocional y despersonalización; sin embargo se encontró diferencias significativas entre los grupos ocupacionales, en relación a la dimensión de baja realización personal, que en mayor porcentaje presentan los médicos. La diferencia solo en la dimensión baja realización personal, ha sido encontrado en el personal de enfermería de hospitales nacionales Arzobispo Loayza y Dos de Mayo de Lima (Arauco, Enciso y García, 2004; Mercado, 2006). 
Factores Sociodemograficos Intra y Extra Organizativos Relacionados con Sindrome de Quemarse por el Trabajo en Profesionales de Salud de Hospitales. Ancash. Perú. 2011.

Leiter y Maslach (1988) y Gil-Monte y Peiró (1997), sustentan la relevancia de la calidad de relaciones interpersonales desarrolladas por los jefes y subordinados en el trabajo, y de ser estas negativas influirían en el desarrollo de desgaste profesional. El predominio de relaciones interpersonales formales necesarias para realizar las tareas en las instituciones laborales, incrementaría los sentimientos de agotamiento emocional y las relaciones interpersonales informales negativas, incrementaría los sentimientos de baja realización personal en el trabajo. En esta investigación se ha encontrado, que existe tanto falta de cohesión dentro de los equipos de trabajo (en muchas ocasiones estos equipos son multidisciplinarios), como conflictos interpersonales con los compañeros de trabajo por chismes y rumores falsos, que son generadores significativos de estrés laboral.

Con respecto a la relación entre SQT y los factores sociodemográficos, los resultados hallados demuestran que el SQT en la fase final ha sido homogéneo, es decir no estaría asociado significativamente a las variables de sexo, edad y estado civil; sin embargo existirían diferencias significativas entre varones y mujeres con respecto a la dimensión baja realización personal, siendo mayor el porcentaje para los varones, esta diferencia no se presento en las dimensiones de cansancio emocional, ni despersonalización. Esto se explicaría por la socialización diferencial en el desarrollo de roles masculinos y femeninos, en el contexto peruano se espera, que los varones logren mayor éxito profesional, económico, político y social.

En otras investigaciones realizadas en el Perú, en grupo de médicos y enfermeras, se han encontrado resultados similares que corroboran los resultados consignados en el párrafo anterior. (Arauco, Enciso y García, 2004; Dávalos, 2005; Quiroz y Saco, 2003). Asimismo otro estudio realizado en personal de enfermería mexicana, refiere que las variables sociodemográficas no presentan ninguna influencia en el SQT. (Leda, Palomera y Morante, 2007). En referencia al tercer objetivo especifico, se correlacionaron los factores intraorganizativos de tipo individual, grupal y organizativo propiamente dicho, con el SQT fase final de los médicos, enfermeros (as) y obstetras y no se ha encontrado diferencias significativas, para el factor individual (tiempo de servicio, horas adicionales semanales laboradas en el hospital, horas semanales laboradas fuera del hospital, horas semanales en ejecución de tareas domesticas, satisfacción laboral, motivación laboral, conflicto de roles, ambigüedad de roles, estado emocional generado en el trabajo) y organizativo (distribución de turnos, asignación de tareas y metas en tiempos prefijados, tipo de liderazgo de jefes de servicio y director del hospital), es decir la existencia de los factores citados ha sido homogéneo, según el hospital en el cual trabajan los profesionales de la salud encuestados; pero para el caso del factor grupal (percepción de apoyo socioemocional en el centro de trabajo, conflictos interpersonales con compañeros de trabajo, conflictos gremiales), se encontró que esta relacionado significativamente con el SQT fase final de los profesionales de la salud evaluados.

En relación al factor grupal, el mayor porcentaje de profesionales de la salud señala que recibe apoyo de tipo informativo y emocional (66.9\%); que mayormente los 
conflictos interpersonales con los compañeros de trabajo son por chismes y rumores falsos, poco trabajo en equipo y escasa cooperación (76.4\%); en menor porcentaje, los encuestados perciben que los conflictos gremiales, generan alta tensión (33.8\%). Este hallazgo es congruente con lo sustentado en la literatura científica revisada (Gil-Monte y Moreno-Jiménez, 2007). De los resultados expuestos, se infiere que el tipo de cultura organizacional predominante (conductas, costumbres y valores practicados por los profesionales de la salud) en los hospitales de la zona sierra de Ancash, esta condicionando el desarrollo, cronicidad y contagio del SQT alto, y también constituiría un factor de riesgo intraorganizativo.

EL SQT fase final presente en los hospitales muestreados, seria un indicador del predominio de un sistema organizacional mixto, con liderazgo participativo, negligente y autocrático, en el cual el tipo de clima laboral seria en parte de desconfianza, temor, inseguridad, poco compromiso e identificación de los profesionales de la salud con la institución laboral y sus objetivos; con una cultura y dinámica organizacional, reflejo de la cultura de la sociedad peruana y como tal y de acuerdo a los resultados hallados, sus características serian: desacuerdo con los bajos salarios percibidos con relativa satisfacción laboral; baja motivación laboral por falta de reconocimiento a la labor desempeñada y ninguna o poca oportunidad de promoción de desarrollo en el ámbito laboral; frecuentes conflictos de roles por ordenes contradictorias impartidas por jefes inmediatos, que mayormente se contradicen con deseos, valores y metas del personal; conductas disfuncionales como, chismes, rumores falsos, poco trabajo en equipo, escasa cooperación entre compañeros de trabajo; conflictos gremiales; rotación de servicio inadecuado; turnos rotativos mal programados; asignación de tareas y metas para ser cumplidos en horarios y tiempos prefijados reducidos.

Asimismo la cultura y dinámica organizacional descrita, es causa y efecto, de una parte afectan la conducta, creencias, valores y actitudes de los profesionales de la salud, y de otra es afectada por factores como, su historia, políticas de gobierno central y regional, legislación laboral, idiosincrasia de la sociedad peruana (falta de unión, baja autoestima, mediocridad, deshonestidad, viveza), cambios científicos tecnológicos, sus recursos humanos que perciben estresores laborales como: no disponer de recursos materiales y equipos, ni espacio laboral suficiente y cómodo (ambientes antiguos no mejorados, ni renovados desde su construcción, décadas 60 y 70), y las características de la tarea, como atender a pacientes con enfermedades infectocontagiosas y críticos (que generan diferentes grados de implicación física y emocional), en ambientes contaminados con desinfección y limpieza inadecuados, relaciones interpersonales negativas entre compañeros de trabajo.

Complementando, se ha encontrado evidencia de diferencias significativas en los puntajes de las dimensiones de agotamiento y despersonalización, asociado al factor intraorganizativo; es decir el personal de salud que refirió la presencia de factores intraorganizativos en su centro laboral, presento mayor agotamiento o cansancio emocional y despersonalización. Este hallazgo es coherente con las tendencias encontradas en investigaciones internacionales (Albaladejo et al, 2004; Palmer et al, 
Factores Sociodemograficos Intra y Extra Organizativos Relacionados con Sindrome de Quemarse por el Trabajo en Profesionales de Salud de Hospitales. Ancash. Perú. 2011.

2005; Albieri y Do Carmo, 2009), nacionales (López, Zegarra y Cuba, 2006; Quiroz y Saco, 2003) y regionales (Lugo, 2010; Flores y Cueva, 2011), como la literatura científica revisada. (Gil-Monte, 2006; Gil-Monte y Moreno-Jiménez, 2007).

En referencia al cuarto objetivo especifico, no se ha encontrado que los factores extraorganizativos (número de horas semanales en actividades de recreación y esparcimiento, actividades en horas libres y vacaciones, conflictos familiares con pareja e hijos, apoyo socioemocional de familiares y amistades), estén relacionados significativamente con el SQT (fase final) de los médicos, enfermeros (as) y obstetras. El resultado descrito en parte coincide con lo encontrado por Quiroz y Saco (2003), referente a que existía baja asociación estadística entre el SQT y las horas semanales laboradas dentro y fuera del hospital, como con las horas de esparcimiento semanal. Al respecto Silveira y Rodríguez-Carvajal, (2007) en su estudio de estrés laboral y síndrome de burnout realizado en profesionales de la salud de Uruguay, no encontraron correlación significativa entre la dimensión baja realización personal e interferencia familiar, ni productividad laboral, solo encontraron correlación entre la dimensión cansancio emocional e interferencia familiar, exclusivamente para el personal de enfermería. Finalmente en este estudio el $40.5 \%$ de encuestados, refieren que los motivos para agredir o discutir con su pareja es la dificultad para conciliar la vida laboral y familiar, como la escasa cooperación de la pareja para realizar tareas domesticas, lo cual indicaría que la interacción entre el contexto laboral y extralaboral (calidad de vínculo familiar, conflictos familiares con la pareja e hijos) incidirían en la aparición y desarrollo del SQT.

\section{CONCLUSIONES}

1. No existe asociación significativa entre los factores sociodemográficos, intra y extraorganizativos, con el SQT (todas sus fases); pero si hay relación significativa entre el factor intraorganizativo grupal (apoyo socioemocional, conflictos interpersonales con compañeros de trabajo, conflictos gremiales) y la fase final de SQT de los médicos, enfermeros (as) y obstetras nombrados, que laboran en cinco hospitales de la zona sierra de Ancash.

2. Se confirmo parcialmente la primera hipótesis específica. El 78.0\% de profesionales de la salud encuestados, presentaron SQT (fase inicial, etapas I y III), siendo significativo la presencia de SQT (fase inicial) para los obstetras del hospital Víctor Ramos Guardia de Huaraz, y de otra se confirmo, que los profesionales de la salud investigados, parcialmente presentan SQT en la etapa final en $20 \%$. Los médicos presentaron (20.0\%) de SQT final, el grupo de enfermeros (as) (21.1\%) y obstetras $(12.5 \%)$. Sin embargo las diferencias estadísticamente no han sido significativas.

3. Existe evidencias de diferencias significativas entre las fases inicial, intermedia y final del SQT en profesionales de salud, según las etapas de Golembiewski.

4. Existe evidencia de diferencias significativas entre los grupos ocupacionales, en relación a la dimensión de baja realización personal, que en mayor porcentaje presentaron los médicos. 
5. Existe evidencia de diferencias significativas entre varones y mujeres, con respecto a la dimensión de baja realización personal $(\mathrm{p}<0.05)$, que es mas frecuente en los varones.

6. Hay evidencia de diferencias significativas en los puntajes de agotamiento emocional y despersonalización, según la presencia del factor intraorganizativo. Los profesionales de la salud, que refieren la presencia de factores intraorganizativos en su trabajo, presentaron mayor agotamiento o cansancio emocional y despersonalización, que aquellos que no refieren $(\mathrm{p}<0.05)$.

7. Se concluye que el SQT (diferentes fases) presente en la muestra evaluada, es un problema de salud pública, ocupacional y mental de índole estructural, propio del entorno y contexto laboral investigado, resultado de la propia naturaleza del trabajo y de una combinación de estresores laborales y sociales, que interactúan con las características psicobiológicas de los profesionales de la salud y que afectan la productividad y calidad de servicio en el trabajo, como en el desarrollo integral de la zona sierra de Ancash.

\section{REFERENCIAS BIBLIOGRAFICAS}

Albaladejo, R., Villanueva, R., Ortega, P., Astasio, P., Calle, ME., Domínguez, V. (2004). Síndrome de Burnout en el personal de enfermería de un Hospital de Madrid. Revista Española de Salud Pública, 78, 004, 505-516. Recuperado de: http://redalyc. uaemex.mx/src/inicio/ArtPdfRed.jsp?iCve $=17078408$

Albieri, D. y Do Carmo M. (2009). Síndrome de Burnout en trabajadores de enfermería del servicio de emergencia de un hospital universitario. Acta Paul Enferm, 22 (2), 192 - 7. Recuperado de: http://www.scielo.br/pdf/ape/v22n2/en_a12v22n2.pdf

Aragón M., Morazan D. y Pérez R. (2008). Síndrome de Burnout en médicos y personal de Enfermería del Hospital Escuela Oscar Danilo Rosales Agüello, León, 2007,Universitas, 2,2, 33-38. Recuperado de:http://www.unanleon.edu.ni/ universitas/pdf/volumen2_nro2/arto6.pdf

Aranda, C., Pando, M., Salazar, J., Torres, T., Aldrete, M:G., Pérez, M.B. (2005). Síndrome de Burnout en médicos familiares del Instituto Mexicano del Seguro social, Guadalajara, México. Revista Cubana Salud Publica, 31(2). Recuperado de: http://hinari-gw.who.int/whalecomscielo.sld.cu/whalecom0/scielo. php?script $=$ sci arttext\&pid =S0864-34662005000200005\&lng =en\&nrm =iso\&tlng =es

Arauco, G., Enciso, J. y García, R. (2004). Factores Sociodemograficos y Síndrome de Burnout en el Profesional de Enfermería del Hospital Nacional Arzobispo Loayza-2004. Tesis para optar el Título de Licenciada en Enfermería. Universidad Peruana Cayetano Heredia. Perú.

Arauco, G. (2007). Síndrome de Burnout en Enfermeras del Hospital Nacional Especializado "Víctor Larco Herrera", 2007. Tesis para optar Licenciatura en Enfermería. Universidad Nacional Mayor de San Marcos. Perú.

Coronado, L. (2006). Factores laborales y niveles de estrés laboral en Enfermeros de los servicios de áreas críticas y medicina del Hospital Nacional Daniel A. Carrión. 
Factores Sociodemograficos Intra y Extra Organizativos Relacionados con Sindrome de Quemarse por el Trabajo en Profesionales de Salud de Hospitales. Ancash. Perú. 2011.

Tesis para optar Licenciatura en Enfermería. Universidad Nacional Mayor de San Marcos. Perú.

Dávalos, H. (2005). Nivel de estrés laboral en enfermeras del Hospital Nacional de Emergencias José Casimiro Ulloa. Tesis para optar Licenciatura en Enfermería. Universidad Nacional Mayor de San Marcos. Perú.

Flores, R. y Cueva, F. (2011). Factores asociados al nivel del síndrome de burnout. En enfermeros del hospital Víctor Ramos Guardia. Huaraz. 2011. (Tesis de licenciatura). Universidad Nacional Santiago Antúnez de Mayolo, Huaraz.

Gálvez, M., Moreno-Jiménez, B. y Mingote, J. (2009). El desgaste profesional del medico: revisión y guía de buenas prácticas. El vuelo de Ícaro. España: Ediciones Díaz de Santos.

Gamonal, Y., García, C. y Silva, Z. (2008). Síndrome de Burnout en el profesional de Enfermería que labora en áreas críticas. Revista Enfermería Herediana, 1(1), 33-39. Recuperado de: http://www.upch.edu.pe/famed/rmh/reh/1-1/v1n1ao5.pdf

Gil-Monte, P. y Peiró, J.M. (1997). Desgaste psíquico en el trabajo: el síndrome de quemarse. Madrid: Síntesis.

Gil-Monte, P. y Moreno-Jiménez, B. (2007). El síndrome de estar quemado por el Trabajo (Burnout). España: Ediciones Pirámide.

Golembiewski, R. T., Munzenrider, R. y Carter, D. (1983). Phases of progressive burnout and their work site covariants: Critical issues in OD research and praxis. Journal of Applied Behavioral Science, 19(4), 461-481.

Golembiewski, R.T., Munzenrider, R.F. y Stevenson, J. (1986). Stress in organizations. NuevaYork: Ed.Praeger.

Hospital Nacional Arzobispo Loayza. (2010). Archivos Estadísticos de la Oficina de Personal. Lima: Autor.

Grau A., Flichtentrei D., Suñer R., Prats, M., y Braga, F. (2009). Influencia de factores personales, profesionales y transnacionales en el síndrome de burnout en personal sanitario hispanoamericano y español, 2007. Revista Española de Salud Publica, 83(2), 215-230. Recuperado de: http://scielo.isciii.es/scielo.php?pid=S1135$7272009000200006 \&$ script $=$ sci_arttext

Leiter, M.P. y Maslach, C. (1988). The impact of interpersonal environment on burnout and organizational commitment.Journal of Organizational Behavior, 9, 297-308

Leda, R.M., Palomera, A. y Morante, M.E. (2007). El síndrome de Burnout en profesionales de enfermería mexicanos. En: Gil-Monte P, Moreno-Jiménez B, coordinadores: $E l$ síndrome de quemarse por el trabajo (Burnout). Grupos profesionales de riesgo, p. 163-170. Madrid: Pirámide.

López, C., Zegarra, A. y Cuba, V. (2006).Factores asociados al síndrome de burnout en enfermeras de emergencia del Hospital Nacional Guillermo Almenara Irigoyen. Revista de Ciencias de la Salud, 1, 1, 53-61. Recuperado de https://docs.google.com/ viewer? $\mathrm{a}=\mathrm{v} \& \mathrm{q}=$ cache:xpcJcpF6K4cJ:posgrado. upeu.edu.pe/revista/file/55-63.pdf 
Lugo, R. (2010). Factores de riesgo asociados al sindrome de burnout en los enfermeros de los hospitales ESSALUD y Víctor Ramos Guardia- Huaraz.2008. (Tesis de maestría). Universidad Nacional Santiago Antúnez de Mayolo, Huaraz.

Llaja, V. (2002). Síndrome de Burnout en el Departamento de Cuidados Intensivos del Hospital Nacional Edgardo RebagliatiMartins- Essalud. Recuperado de: http://bvs. org.pe/cgi-bin/wxis.exe.iah

Maslach, C. (1982). Burnout: A social psychological analysis. En J.W.Jones (Ed.), The burnout syndrome: current research, theory, interventions (pp.30-53). Park ridge, Illinois: London House Press.

Maslach, C. y Jackson, S.E. (1997). MBI Inventario"Burnout" de Maslach. Síndrome de quemado por estrés laboral asistencial. Madrid: TEA Ed. S.A.

Melita A., Cruz, M. y Merino, J. (2008). Burnout en profesionales de enfermería que trabajan en centros asistenciales de la octava región, Chile. Ciencia y Enfermería, 14 (2), $75-85$.

Mercado, G. (2006). Nivel de Estrés laboral en enfermeras en el Hospital Nacional Dos de Mayo, enero 2006. Tesis para optar Licenciatura en Enfermería. Universidad Nacional Mayor de San Marcos. Perú.Palmer, Y., Gómez, A., Cabrera, C., Prince, R. y Searcy, R. (2005). Factores de riesgo organizacionales asociados al síndrome de burnout en médicos anestesiólogos. Salud Mental, 28 (001),82-91.

Peralta, E. y Vargas, J. (2009). Agotamiento emocional en el personal de enfermería del Hospital de Niñez Oaxaqueña: la prevención del Burnout.Centro Regional de Investigación en Psicología, 3(1), 5-10. Recuperado de: http://www.conductitlan. net/42_agotamiento_emocional_burnout.pdf

Quiroz, R. y Saco, S. (2003).Factores Asociados al Síndrome de Burnout en médicos y Enfermeras del Hospital Nacional Sur Este de EsSalud del Cusco. Revista SITUA Semestral de la Facultad de Medicina Humana, 11 - 22, Recuperado de: http://www. opas.org.br/gentequefazsaude/bvsde/bvsacd/cd49/a04.pdf

Silveira, N. y Rodríguez-Carbajal, R. (2007). ). El estrés laboral, la satisfacción laboral y el síndrome de burnout en profesionales de la salud de Uruguay. En: Gil-Monte P, Moreno-Jiménez B, coordinadores: El síndrome de quemarse por el trabajo (Burnout). Grupos profesionales de riesgo, p.171-185. Madrid: Pirámide.

Tapia, T., Avalos M., Vargas, R., Gómez, A. y Rodríguez, A. (2009). Síndrome de Burnout en Enfermeras de un Hospital Regional. Rev. Enferm. Inst. Mex. Seguro Soc, 17(1), 31 - 38. Recuperado de: http://www.medigraphic.com/pdfs/enfermeriaimss/ eim-2009/eim091f.pdf 